\title{
An interesting case of atypical venous thrombosis with low red blood cells
}

\author{
Giuseppe Chiariello, Raimondo Cavallaro, Giuseppe Covetti, Maria Carla Attilia Pisano, Antonella Schettini, \\ Tiziana D’Aniello, Rossella Nappo, Emilio Aliberti, Arcangelo Iannuzzi
}

Department of Medicine and Medical Specialties, Section of Internal Medicine 2, Antonio Cardarelli Hospital, Naples, Italy

\begin{abstract}
Sex: F Age: 65 years. Recent abdominal colic. Petechial-like manifestations with painful discolored skin lesions (suggestive of dermal veins thromboses) on the chest and abdomen. Blood levels of glucose, electrolytes, amylase, lipase, total protein, renal-function tests, the prothrombin time, the international normalized ratio, the partial-thromboplastin time and albumin were normal. Laboratory testing revealed hemolytic anemia: red cells $2.94 \times 10^{6} / \mathrm{UL}$; hemoglobin $8.9 \mathrm{~g} / \mathrm{dL}$; lactate dehydrogenase $950 \mathrm{IU} / \mathrm{L}$. Computed tomography of the abdomen and pelvis performed after the administration of intravenous contrast material, revealed a central filling defect in the hepatic veins and their branches that was compatible with acute thrombosis of the hepatic veins and their branches. It was suspected paroxysmal nocturnal hemoglobinuria for which flow cytometric analysis was requested.
\end{abstract}

\section{Case Report}

Sex: F born in Naples. Age: 65 years. Diagnosis on admission: suspected vasculitis.

History: Admitted to Emergency Department with cutaneous rash and abdominal pain. Patient came to ward from short-stay observation unit. She reported recent abdominal colic, and taking a prescribed antibiotic, from which she said that had developed an

Correspondence: Arcangelo Iannuzzi, Department of Medicine and Medical Specialties, Antonio Cardarelli Hospital, via A. Cardarelli 9, 80131 Naples, Italy.

Tel.: +39.081.7472121 - Fax: +39.081.7472121.

E-mail: arcangelo.iannuzzi@aocardarelli.it

Key words: Thrombosis; anemia; hemolysis.

Contributions: GC, AI, study concept and design; GC, RC, GC, MCAP, AS, TD'A, RN, management, analysis, and interpretation of the clinical case; EA, AI, drafted or critically revised the manuscript for important intellectual content. All authors read and approved the final manuscript.

Conflict of interests: the authors declare no potential conflict of interests.

Received for publication: 15 September 2019.

Accepted for publication: 7 October 2019.

This work is licensed under a Creative Commons Attribution NonCommercial 4.0 License (CC BY-NC 4.0).

${ }^{\circ}$ Copyright: the Author(s), 2019

Licensee PAGEPress, Italy

Italian Journal of Medicine 2020; 14:22-26

doi:10.4081/itjm.2019.1212 allergic skin reaction. Previous seizures for which she is now taking levetiracetam $500 \mathrm{mg}$ oral once nightly. Awake and collaborative, eupneic at rest. No chest pain. Blood pressure $125 / 70 \mathrm{mmHg}$, heart rate 88 bpm, $\mathrm{SpO}_{2} 93 \%$ in air. Apyrexial. Vesicular murmur heard on auscultation of the chest. Soft and diffusely tender abdomen with no rigidity or guarding. No ankle swelling. Evidence of recent petechial-like manifestations with painful discolored skin lesions (suggestive of dermal veins thromboses) on the chest and abdomen.

Blood tests: urea $42.0 \mathrm{mg} / \mathrm{dL}$; creatinine 0.76 $\mathrm{mg} / \mathrm{dL}$; total bilirubin $0.45 \mathrm{mg} / \mathrm{dL}$; direct bilirubin 0.1 $\mathrm{mg} / \mathrm{dL}$; indirect bilirubin $0.3 \mathrm{mg} / \mathrm{dL}$; lactate dehydrogenase $950 \mathrm{IU} / \mathrm{L}$; ferritin $893 \mathrm{ng} / \mathrm{mL}$; red cells $2.94 \times 10^{6} / \mathrm{UL}$; hemoglobin $8.9 \mathrm{~g} / \mathrm{dL}$; white cells $9.05 \times 10^{3} / \mathrm{UL}$; platelets $221 \times 10^{3} / \mathrm{UL}$; haptoglobin 0.08 $\mathrm{g} / \mathrm{L}$; anti-nuclear antibodies (ANA)-titer: present 1:160; ANA-pattern: speckled; anti dsDNA Ab 3.0 (negative); ENA negative; negative indirect antiglobulin test; direct Coombs test was negative.

US abdomen: liver, pancreas and spleen free from focal lesions. Biliary tract not dilated. Gall bladder with mostly dense biliary contents. No signs of urinary stasis. Empty urinary bladder. Diffuse distension of the colon with fecal impaction. No evidence of collections in the peritoneal cavity.

Computed tomography (CT) thorax with contrast: no focal changes of lung parenchyma. No focal changes of pleural lining. No mediastinal or peribronchial lymphadenopathy. Regular opacification of the pulmonary trunk and main branches of the pulmonary artery. Trachea and bronchi patent. No evidence of pleural effusion. Nodular hyperplasia of the thyroid with expansion into the mediastinum. 
CT abdomen and pelvis with contrast: no evidence of peritoneal effusion. Liver within normal limits, homogenous density of parenchyma with no evidence of focal lesions. Biliary vessels not dilated. Patent portal vein and splenic vein. The presence of non-occluding thrombotic-like defects in the hepatic veins and their branches is noted. Patent superior vena cava and inferior vena cava. Spleen, pancreas and adrenal glands within normal limits of size and volume with homogenous density. Kidneys in situ, within normal limits of size and volume, with regular enhancement post-contrast. Renal pelvis not dilated. Bilateral renal cysts (25 $\mathrm{mm})$. Empty bladder. No evidence of lymphadenopathy in the coeliac, lumbar-aortic, iliac, inguinal and obturator nodes. Retroperitoneal vessels follow regu- lar course and are of normal size with normal opacification of lumen.

The presence of venous thrombosis in abnormal sites (hepatic and dermal veins) in a patient with no hepatic pathology, together with abdominal pain and intravascular hemolysis [increased lactate dehydrogenase (LDH), negative Coombs anemia, reduced haptoglobin], even in the absence of evident hemoglobinuria, has led us to suspect paroxysmal nocturnal hemoglobinuria (PNH) (Table 1) for which flow cytometric analysis was requested (Figure 1).

Discharge diagnosis: paroxysmal nocturnal hemoglobinuria complicated by hepatic vein thrombosis. Nodular hyperplasia of the thyroid with mediastinal expansion in euthyroid patient.

Table 1. When to test for paroxysmal nocturnal hemoglobinuria.

\footnotetext{
1. Intravascular hemolysis (hemoglobinuria)
}

2. Unexplained hemolysis + (see next column)
Hyposideremia

Abdominal pain

Atypical venous thromboses

Granulocytopenia/Thrombocytopenia

3. Acquired hemolytic anemia with negative Coombs, in the absence of schistocytes and in the absence of infective disease

4. Atypical thromboses, see next column (especially if accompanied by hemolytic anemia or cytopenia)

Hepatic veins (Budd-Chiari)

Portal vein and splenic vein (in absence of cirrhosis)

Cerebral veins

Dermal cutaneous veins

5. Marrow aplasia (aplastic or hypoplastic)

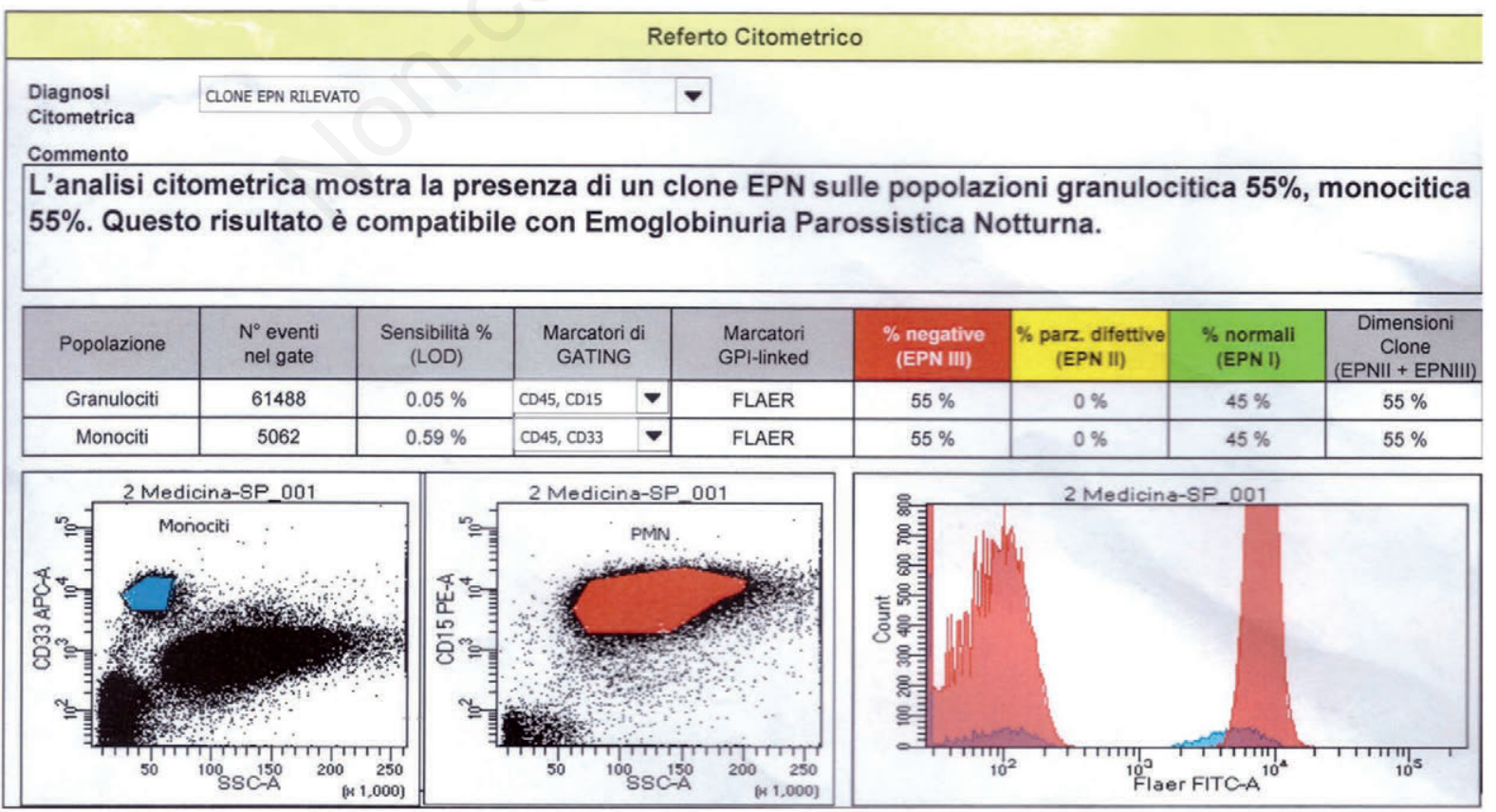

Figure 1. Flow cytometric analysis. 


\section{Discussion}

$\mathrm{PNH}$ is a condition of clonal origin from multipotent hematopoietic stem cells characterized by anemia, bone marrow failure and thrombophilia due to an acquired mutation of the PIGA gene (Xp22.1), in which there is a reduced synthesis of the glycosylphosphatidylinositol (GPI) anchor on the hematopoietic stem cell membrane. ${ }^{1}$ According to the escape theory, the PIGA gene undergoes a series of somatic mutations in hematopoietic stem cells, in a two-step process, which determines clonal expansion. ${ }^{2}$ The PIGA gene produces a glycosyl transferase protein which is an essential component of the biosynthetic pathway that produces GPI. Following somatic mutation of the PIGA gene, a protein is formed with partial or complete loss of enzymatic function, resulting in an almost complete absence of GPI-anchored proteins. Among the cellular membrane constituents which are GPI-anchored, there are the CD55 complement inhibitory proteins [decay accelerating factor (DAF)] and CD59 [membrane inhibitor of reactive lysis (MIRL)]. Deficiency of these two proteins results in complement mediated intravascular hemolysis. Although the loss of GPI-anchored proteins affects all cells derived from hematopoietic stem cells (erythrocytes, granulocytes, monocytes, lymphocytes, and platelets), only CD55 and CD59 deficiency contributes clinically to the pathophysiology of PNH with complement-mediated intravascular hemolysis. The relationship between deficits in CD55 and CD59 and other manifestations of PNH, such as medullary aplasia and thrombophilia, is not well known. Prevalence is not well known due to little available data but the prevalence of patients with $\mathrm{PNH}$ clones is estimated to be 15 per million, ${ }^{3}$ whereas the incidence is 1 in a million in the total population. ${ }^{4}$ There is no difference in incidence between the sexes and the median age of diagnosis is just over thirty years, even if there are documented cases in children, adolescents and older adults. ${ }^{5}$ The pathophysiology of intravascular hemolysis in PNH is due to the fact that normal red blood cells are protected from the attack of complement by GPI-anchored proteins CD55 and CD59, while in PNH red blood cells are not protected by these anchored GPI proteins and are lysed by complement, giving rise to hemoglobinemia and anemia. Intravascular hemolysis also leads to a rise in LDH levels, indirect bilirubin and reticulocytes, and to a reduction in haptoglobin levels. As it develops intravascularly, hemolysis favors the development of thrombotic phenomena. Hemoglobinemia favors the depletion of nitric oxide (NO), presenting as abdominal pain, dysphagia, sexual dysfunction, and pulmonary hypertension. Hemoglobinuria can result in renal damage, both as tubular toxicity and interstitial nephritis with subsequent acute and/or chronic renal insufficiency. Psycho-somatic implications are also important in this condition, with chronic fatigue, apathy, malaise, and perception of a poor quality of life in the majority of patients suffering from this disease. The clinical manifestations of this disease are varied, with some patients developing a pathology predominantly characterized by complement mediated hemolysis while others predominantly display signs of medullary aplasia with modest signs of intravascular hemolysis. Clinical heterogeneity, although not completely understood, could be due to the interrelations between PNH and immune-mediated medullary aplasia, taking into account that $\mathrm{PNH}$ is an acquired, nonmalignant, clonal hematopoietic stem cell disease. Understanding the pathophysiology which is unique both to the hemolytic action of the complement and to the immune-mediated medullary aplasia provides the basis for the correct management of the disease. The two main aspects which characterize $\mathrm{PNH}$ and distinguish it from other hemolytic anemias are primarily the involvement of hematopoietic stem cells, not only of the red blood cell, and also, unlike other intrinsic anomalies of erythrocytes, PNH is an acquired and nonhereditary disease. Early diagnosis can avoid clinical complications possibly created by the typical diagnostic delay that accompanies patients suffering from rare diseases, improving management and their quality of life. ${ }^{6}$

For rare diseases, exploring and intercepting cases before they are overtly apparent is a challenge, with the aim being to provide precise and accurate care as soon as possible. ${ }^{7}$ Presently the gold standard for obtaining a definitive diagnosis of $\mathrm{PNH}$ is flow cytometry with antigens that bind to the GPI protein. The diagnosis of PNH is straightforward when flow cytometric analysis of the peripheral blood reveals a population of glycosyl phosphatidylinositol anchor protein-deficient cells. ${ }^{8}$ Until a few years ago, the only curative treatment option for $\mathrm{PNH}$ was allogenic transplant, which was burdened with a high morbidity and mortality. ${ }^{9} 10$ Symptomatic palliative treatment was confined to non-targeted therapy with blood transfusions, corticosteroids, oral anticoagulants or heparin in the case of thrombotic events. Currently, however, we have specific monoclonal antibodies (eculizumab) that binds with high affinity to $\mathrm{C} 5$, so that the terminal complement activity is blocked while the proximal complement functions remain intact. In the TRIUMPH study eculizumab led to a complete reduction in the number of transfusions received and a reduction of LDH to normal concentrations, with a net improvement in the relative score of asthenia. ${ }^{11}$ This drug also results in an $85 \%$ reduction of thrombotic events and an improvement in renal function with reduced damage in patients with renal damage caused by hemolysis. In 2003 eculizumab was designated an orphan drug in Europe, in 2007 it was inserted onto List 648 in Italy for the treatment of patients with $\mathrm{PNH}$, and in 
2008 a decree regarding reimbursement and sales price (high) in Italy (AIFA register) was published. Side effects are rarely clinically significant (nasopharyngitis, urinary tract infections, upper airways infections, dizziness, headache, nausea, diarrhea).

\section{Conclusions: the 5 key questions about paroxysmal nocturnal hemoglobinuria}

\section{Is paroxysmal nocturnal hemoglobinuria a neoplastic disease?}

No, PNH is a disease of clonal cells in which mutation of the PIGA gene is used as a marker of clonality, but it is not a neoplastic disease like chronic myeloid leukemia or acute myeloid leukemia in which clone cells mutate and expand indefinitely until a specific therapy is not started. Also, in PNH expressivity of the clone changes from case to case: in some cases PNH is hardly appreciable at flow cytometric analysis (as in the subclinical form), sometimes it is fairly well expressed (as in the form that accompanies medullary aplasia), in other cases it is widely represented, being expressed in most hematopoietic stem cells (classical form of PNH); in no case PNH invades other organs and tissues outside the bone marrow.

\section{Does flow cytometric analysis only reveal mutated clones on red blood cells?}

No, it is detectable in all cells derived from hematopoietic stem cells (erythrocytes, granulocytes, monocytes, lymphocytes and platelets): an accurate diagnosis requires that flow cytometry is performed not only on red blood cells (many are destroyed by intravascular hemolysis and others are potentially coming from blood transfusions) but also on polymorphonuclear cells, in particular granulocytes and monocytes, which are not lysed by the membrane attack complex (MAC). Today flow cytometry is the gold standard for the diagnosis and monitoring of $\mathrm{PNH}$ and is the most sensitive method to reveal cells that lack GPI anchoring proteins. Modern flow cytometers are currently capable of analyzing at least 6 fluorescent parameters or antigens on a routine basis. This increase in the level of sophistication and complexity means that small populations of abnormal cells can be easily discovered, although in some circumstances the clinical significance of results is not fully understood. Although CD55 and CD59 are widely used to find abnormal granulocyte clones, there are data suggesting that these reagents are not as valid as other antibodies and antigens for the diagnosis of $\mathrm{PNH} .{ }^{12}$ Recent data suggest that one of the best available agents to study antigens bound to GPI on leukocytes is fluorescent aerolysin or FLAER. ${ }^{13}$ This is an inactive variant conjugated to the fluorochrome of the aerosolized protein which is bound specifically to GPI anchors and will therefore be absent from granulocytes and monocytes deficient in GPI anchors (PNH cells). FLAER has significant advantages as a reagent in the diagnosis of PNH since, unlike antibodies against the GPI-linked antigens normally studied, its binding is less affected by the maturity of the cell. Also, it can be used in multicolor combinations with monoclonal antibodies directed to both GPI-linked and non-GPI-linked antigens in the detection of EPN clones. ${ }^{14}$ For this reason this method was used in the case.

\section{Why does the mutation of the PIGA gene cause paroxysmal nocturnal hemoglobinuria?}

A normally functioning PIGA gene codes for the synthesis of an enzyme (acetyl glucosamine transferase) which allows the glycation of inositol phosphate (uridine diphosphate $\mathrm{N}$-acetylglucosamine + phosphatidyl inositol $\rightarrow \mathrm{N}$-acetylglucosaminyl phosphatidyl inositol); this is a fundamental initial enzymatic stage in allowing the construction of GPI-anchored proteins. Among the particularly important proteins which bind to GPI, and inhibit hemolytic action via alternative pathway of complement, are DAF (also called CD55) and MIRL (also called CD59). While the reason for intravascular hemolysis is clear in $\mathrm{PNH}$, the pathophysiology related to the occurrence of thrombotic events, another typical characteristic of $\mathrm{PNH}$, is not yet completely understood even though several possible explanations have been proposed. ${ }^{15}$

\section{Which complement pathways provoke intravascular hemolysis in paroxysmal nocturnal hemoglobinuria?}

Chronic intravascular hemolysis is caused by the alternative pathway of complement, a component of innate immunity and an evolved mechanism to protect the host from pathological organisms. This is different from the classical pathway of complement which forms part of acquired immunity and requires a reaction with an antigen to activate; the alternative pathway is always active. The alternative pathway of the complement cascade needs two fundamental components in order to function: amplification of $\mathrm{C} 3$ and $\mathrm{C} 5$ convertases and the activation of the MAC. Safeguarding systems are in place to protect the host from continuous attack by the alternative pathway. In the case of red cells, protection against cell lysis mediated by the alternative pathway, occurs mainly thanks to two proteins: CD55 and CD59, which act on different levels of the complement cascade. CD55 regulates the formation and activity of the $\mathrm{C} 3$ and $\mathrm{C} 5$ convertases, whereas CD59 blocks the formation of MAC, which is composed of $\mathrm{C} 5 \mathrm{~b}, \mathrm{C} 6, \mathrm{C} 7, \mathrm{C} 8$ and many molecules of C9. The absence of CD55 and CD59 on the red 
blood cell membrane represents the distinctive element of clonal mutation in PNH and explains the pathophysiology of complement mediated hemolysis, and also justifies why Coombs testing is negative.

\section{Why is eculizumab effective in treating classical paroxysmal nocturnal hemoglobinuria?}

Eculizumab is a humanized monoclonal antibody which binds to a factor $\mathrm{C} 5$ of complement, inhibiting the activation of $\mathrm{C} 5$ into $\mathrm{C} 5 \mathrm{~b}$ by $\mathrm{C} 5$ convertase in the alternative pathway of complement and blocking the formation of the MAC and, therefore, intravascular hemolysis. Thus, treatment with eculizumab reduces the need for blood transfusions, it improves anemia and the quality of life in patients by reducing the symptomatology associated with chronic hemolysis (malaise, tiredness, fatigue); with treatment bilirubin, haptoglobin and LDH return to normal. Eculizumab does not cure the disease in the sense that red blood cells are still deficient in GP1-anchored proteins and defective cells continue to be produced even if they are not lysed by complement. From a pathophysiologic point of view, the drug should not have an effect on thrombotic complications of the disease and these patients need to continue heparin or oral anticoagulation if they have had a thrombotic event, but there is some evidence from clinical studies that eculizumab reduces the incidence of thrombotic events in these patients, particularly in the Budd-Chiari syndrome. ${ }^{16}$ Finally, because patients who have a deficit in $\mathrm{C} 5$ are more at risk of meningitis, patients in therapy with eculizumab have to be prophylactically vaccinated against meningitis $\mathrm{B}, \mathrm{C}$ and $\mathrm{Y}$.

\section{References}

1. Takeda J, Miyata T, Kawagoe K, et al. Deficiency of the GPI anchor caused by a somatic mutation of the PIG-A gene in paroxysmal nocturnal hemoglobinuria. Cell 1993;73:703-11.

2. Young NS, Maciejewski JP. Genetic and environmental effects in paroxysmal nocturnal hemoglobinuria: this little PIG-A goes “Why? Why? Why?". J Clin Invest 2000; 106:637-41.

3. Hill A, Platts PJ, Smith A, et al. The incidence and prevalence of paroxysmal nocturnal hemoglobinuria (PNH) and survival of patients in Yorkshire. Blood 2006;108:985.

4. Hill A, DeZern AE, Kinoshita T, Brodsky RA. Paroxysmal nocturnal haemoglobinuria. Nat Rev Dis Primers 2017;3:17028.

5. Moyo VM, Mukhina GL, Garrett ES, Brodsky RA. Natural history of paroxysmal nocturnal haemoglobinuria using modern diagnostic assays. Br J Haematol 2004; 126:133-8

6. Risitano AM. Paroxysmal nocturnal hemoglobinuria and other complement-mediated hematological disorders. Immunobiology 2012;217:1080-7.

7. Alfano R, Palladino R, Risitano A, et al. Network references for rare diseases: state of the art for the paroxysmal nocturnal hemoglobinuria Epidemiol Prev 2018;42:333-43.

8. Parker CJ. Update on the diagnosis and management of paroxysmal nocturnal hemoglobinuria. Hematology Am Soc Hematol Educ Program 2016;2016:208-16.

9. Moyo VM, Mukhina GL, Garrett ES, Brodsky RA. Natural history of paroxysmal nocturnal haemoglobinuria using modern diagnostic assays. Br J Haematol 2004; 126:133-8.

10. Marti-Carvajal AJ, Anand V, Cardona AF, Sola I. Eculizumab for treating patients with paroxysmal nocturnal hemoglobinuria. Cochrane Database Syst Rev 2014;10:CD010340.

11. Schubert J, Hillmen P, Röth A, et al.; TRIUMPH Study Investigators. Eculizumab, a terminal complement inhibitor, improves anaemia in patients with paroxysmal nocturnal haemoglobinuria. Br J Haematol 2008; 142:263-72.

12. Brodsky RA, Mukhina GL, Li S, et al. Improved detection and characterization of paroxysmal nocturnal hemoglobinuria using fluorescent aerolysin. Am J Clin Pathol 2000;114:459-66.

13. Sutherland DR, Kuek N, Davidson J, et al. Diagnosing PNH with FLAER and multiparameter flow cytometry. Cytometry B 2007;72B:167-77.

14. Borowitz MJ, Craig FE, DiGiuseppe JA, et al.; on behalf of the Clinical Cytometry Society. Guidelines for the diagnosis and monitoring of paroxysmal nocturnal hemoglobinuria and related disorders by flow cytometry. Cytometry Part B (Clinical Cytometry) 2010;78B:211-30.

15. Hill A, Kelly RJ, Hillmen P. Thrombosis in paroxysmal nocturnal hemoglobinuria. Blood 2013;121:4985-96.

16. Haughton J, Kelly RJ, Richards SJ, et al. Improved outcomes of Budd-Chiari syndrome in paroxysmal nocturnal hemoglobinuria with eculizumab therapy. Blood 2012;120:3478. 